\title{
Article
}

\section{Leaf Transcriptome Analysis of Broomcorn Millet Uncovers Key Genes and Pathways in Response to Sporisorium destruens}

\author{
Fei Jin, Jiajia Liu, Enguo Wu, Pu Yang ${ }^{(}$, Jinfeng Gao, Xiaoli Gao and Baili Feng * \\ State Key Laboratory of Crop Stress Biology in Arid Areas, College of Agronomy, Northwest A\&F University, \\ Yangling 712100, China; 18833033167@163.com (F.J.); ljjzl2014@163.com (J.L.); WEG2990762254@163.com (E.W.); \\ yangpu5532@hotmail.com (P.Y.); gaojf7604@126.com (J.G.); gao2123@nwsuaf.edu.cn (X.G.) \\ * Correspondence: fengbaili@nwafu.edu.cn
}

Citation: Jin, F.; Liu, J.; Wu, E.; Yang, P.; Gao, J.; Gao, X.; Feng, B. Leaf Transcriptome Analysis of Broomcorn Millet Uncovers Key Genes and Pathways in Response to Sporisorium destruens. Int. J. Mol. Sci. 2021, 22, 9542. https://doi.org/10.3390/ ijms22179542

Academic Editor: Massimo Maffei

Received: 7 June 2021

Accepted: 27 August 2021

Published: 2 September 2021

Publisher's Note: MDPI stays neutral with regard to jurisdictional claims in published maps and institutional affiliations.

Copyright: (C) 2021 by the authors Licensee MDPI, Basel, Switzerland. This article is an open access article distributed under the terms and conditions of the Creative Commons Attribution (CC BY) license (https:// creativecommons.org/licenses/by/ $4.0 /)$.

\begin{abstract}
Broomcorn millet (Panicum miliaceum L.) affected by smut (caused by the pathogen Sporisorium destruens) has reduced production yields and quality. Determining the tolerance of broomcorn millet varieties is essential for smut control. This study focuses on the differences in the phenotypes, physiological characteristics, and transcriptomes of resistant and susceptible broomcorn millet varieties under Sporisorium destruens stress. In diseased broomcorn millet, the plant height and stem diameter were reduced, while the number of nodes increased. After infection, the activities of superoxide dismutase and peroxidase decreased, and malondialdehyde and relative chlorophyll content (SPAD) decreased. Transcriptome analysis showed 514 and 5452 differentially expressed genes (DEGs) in the resistant and susceptible varieties, respectively. The Kyoto Encyclopedia of Genes and Genomes (KEGG) enrichment analysis of DEGs showed that pathways related to plant disease resistance, such as phenylpropanoid biosynthesis, plant-pathogen interaction, and plant hormone signal transduction, were significantly enriched. In addition, the transcriptome changes of cluster leaves and normal leaves in diseased broomcorn millet were analysed. Gene ontology and KEGG enrichment analyses indicated that photosynthesis played an important role in both varieties. These findings lay a foundation for future research on the molecular mechanism of the interaction between broomcorn millet and Sporisorium destruens.
\end{abstract}

Keywords: broomcorn millet; stress; Sporisorium destruens; transcriptome; Panicum miliaceum; smut

\section{Introduction}

Smut is a common plant disease [1,2], and is one of the major diseases of broomcorn millet (Panicum miliaceum L.), able to seriously affect production. This disease not only reduces yield, it also leads to the contamination of grains and straws [3]. The incidence is generally $5-10 \%$, although in severe cases it may reach $40 \%$ [4]. Smut is a systemic invasive disease in which the pathogen infects the host at the seedling stage, but obvious symptoms appear at the heading stage and beyond [5]. The pathogens of smut include Basidiomycota species and Sporisorium destruens. After Sporisorium destruens infects broomcorn millet, it ruins the grain harvest, and pathogens attach to the soil or seed to overwinter and become a source of infection. A high-temperature, high-humidity environment is conducive to the invasion of broomcorn millet by Sporisorium destruens [6]. At the early heading stage, diseased broomcorn millet does not show significant differences compared to uninfected plants, and smut prevention and treatment in production is more difficult.

To prevent pathogen invasion, plants have evolved specific immune strategies, including pathogen-associated molecular pattern (PAMP)-triggered immunity (PTI) [7], which identifies pathogen invasion, followed by a host defence response [8,9]. Cytoplasmic $\mathrm{Ca}^{2+}$ concentration has been recognised as an essential signal for PTI [10]: during PTI, host cells perceive PAMP through pattern-recognition receptors; this induces an increase in the concentration of cytosolic $\mathrm{Ca}^{2+}$ and leads to activation of calcium-based defence responses [10]. Stomatal behaviour not only influences the balance between $\mathrm{CO}_{2}$ uptake and water loss, 
it also increases the risk of lethality upon stress; the hypersensitive response (HR) is a common feature of plant immune responses, and a type of programmed cell death [11,12]. $\mathrm{Ca}^{2+}$ is known to affect the HR, as well as cell wall and stomatal changes $[11,13,14]$.

Broomcorn millet, an allotetraploid from China with a long cultivation history [15], is cultivated in Europe, the Middle East, and other regions [16], has high nutritional value, and can be used for food, feed, and medicine [17]. Broomcorn millet is tolerant to drought stress and barren soil, and can serve as a pioneer crop, suitable for sustainable agricultural practices [18]. Although the production of broomcorn millet has many advantages, it is grown on a small scale in only a few regions [19], and head smut threatens this industry by substantially reducing yields. Research on broomcorn millet smut has focused mainly on physiological and biochemical responses, agronomic traits, and the effect on yield [20-22], as well as fungicide screening and resistance identification [23,24]. Furthermore, there are few reports on the breeding of broomcorn millet smut resistance and the plant-pathogen interaction mechanisms that negatively impact on broomcorn millet production and smut research.

The high-throughput capabilities of RNA sequencing (RNA-Seq) technology can be used to evaluate gene transcription levels $[25,26]$ and study the interaction mechanism between plants and pathogens at the molecular level [27]. In this study, we investigated and compared the expression of differentially expressed genes (DEGs) in susceptible and resistant broomcorn millet varieties under Sporisorium destruens stress. The changes in plantpathogen interaction, oxidation-reduction process, and plant hormone signal transduction pathways were analysed. These results provide a valuable reference information for the study of interaction between broomcorn millet and Sporisorium destruens.

\section{Results}

\subsection{Phenotype Analysis of Resistant/Susceptible Broomcorn Millet after Sporisorium} destruens Infection

At the early heading stage (about 40 days after planting) of two broomcorn millet varieties, there was no significant difference between uninoculated controls (CK; R0 and S0) and Sporisorium destruens-inoculated samples (R1 and S1). After the heading stage, the ears at the top of the diseased plant were transformed into small cluster leaves, while other treatments appeared normal (Figure 1A). The plant height and stem diameter of diseased broomcorn millet of both varieties were significantly decreased (Figure 1B,C), and the node number increased (Figure 1D). The differences between varieties were not significant ( $p>0.05$ ), except for the stem thickness of uninoculated plants (R0 vs. S0; $p<0.05$ ), and the node number of inoculated plants (R1 vs. S1; $p<0.05$ ).

\subsection{Redox Status and Relative Chlorophyll Content of Broomcorn Millet Leaves under Sporisorium destruens Stress}

To reveal the physiological responses in the leaves of the two broomcorn millet varieties under Sporisorium destruens stress, we determined the activities of superoxide dismutase (SOD) and peroxidase (POD), as well as malondialdehyde (MDA) and relative chlorophyll content (SPAD) index (Figure 2). The differences in SOD activity, MDA content, and SPAD index showed similar trends, i.e., they were lower in the inoculated leaves than in the uninoculated leaves. In the smut-resistant variety, Bameng Xiaohei mi (BM), POD activity was lower in uninoculated leaves than in inoculated leaves, whereas in the smut-susceptible variety, Nianfeng No. 5 (NF), the POD activity in uninoculated leaves was higher than in inoculated leaves. 
A

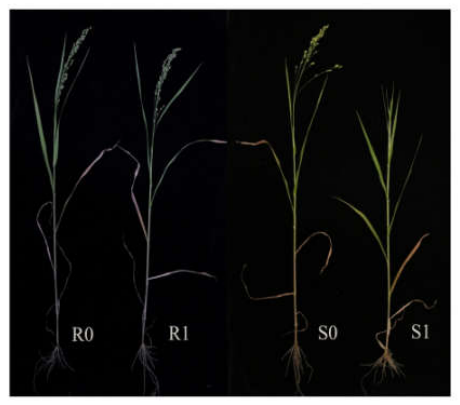

C

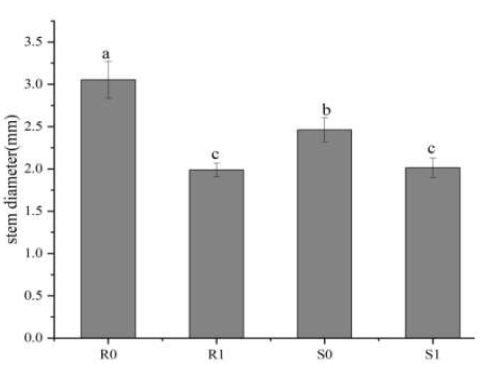

B

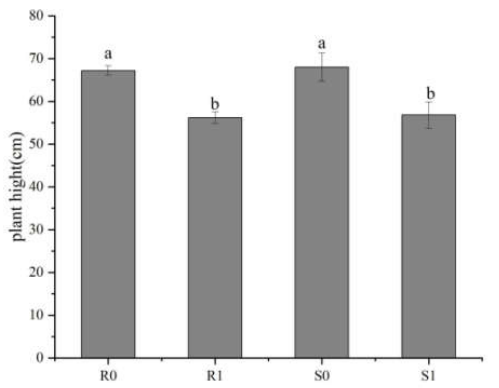

D

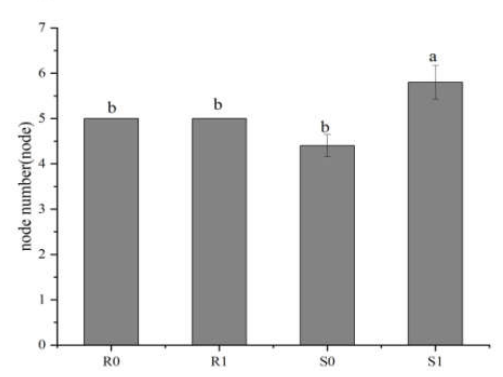

Figure 1. Phenotypic differences between four broomcorn millet samples: R0 and S0 were uninoculated controls; R1 and S1 were inoculated with Sporisorium destruens. (A) Phenotype at heading stage; (B) Plant height (cm); (C) Stem diameter (mm); (D) Node number. The values are presented as means $(n=5)$ with standard deviations. Values with different letters within the same figure were significantly different $(p<0.05)$. Data represents mean \pm SD and different letters $(a, b$, and $c)$ indicate significant differences $(p<0.05)$ in each samples.
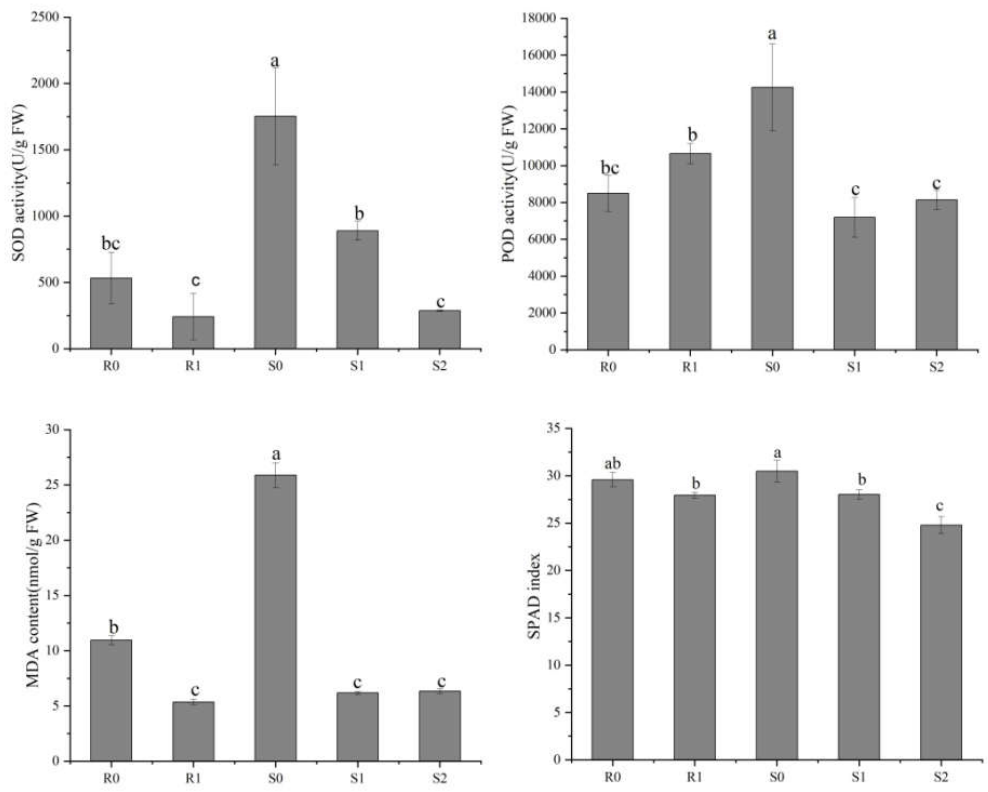

Figure 2. Physiological differences between four broomcorn millet samples. These physiological indices include superoxide dismutase (SOD) activity, peroxidase (POD) activity, malondialdehyde (MDA) content and SPAD index. The values are presented as means $(n=3)$ with standard deviations. Values with different letters within the same figure were significantly different $(p<0.05)$. Data represents mean $\pm \mathrm{SD}$ and different letters $(\mathrm{a}, \mathrm{b}$, and $\mathrm{c})$ indicate significant differences $(p<0.05)$ in each samples, while $\mathrm{ab}$, bc indicate not significant differences $(p>0.05)$. 


\subsection{Data Quality and DEGs in Two Broomcorn Millet Varieties under Sporisorium destruens Stress}

We obtained 15 complementary DNA (cDNA) libraries with high sequencing quality using RNA sequencing (RNA-Seq) (Supplementary Material file 1). Principal component analysis (PCA) clusters samples based on the gene expression of samples, reflecting the repeatability of the samples. The R0-1 sample was identified as an outlier and removed in the following analysis (Figure 3A, Supplementary Material file 2). Venn analysis indicated that there were 30,545 co-expressed genes among the samples; R0, R1, S0, and S1 had 370, 300, 336, and 1686 expressed genes, respectively (Figure 3B). In BM, 514 genes were defined as DEGs in R1 compared with R0, including 28 up-regulated and 486 down-regulated DEGs. In NF, 5452 genes were identified as DEGs, with 3989 up-regulated and 1463 down-regulated. A total of 231 genes were identified as DEGs in the four samples of the two varieties. Compared with CK, the inoculated treatments had higher levels of DEGs. Overall, more DEGs were characterised in NF than in BM (5452 vs. 514, respectively; 10.6-fold difference). In NF, most of the DEGs (3989 out of 5452; 73.17\%) were up-regulated, and 1463 DEGs were down-regulated; however, only 28 DEGs (accounting for 5.4\%) were characterised as being up-regulated in $\mathrm{BM}$, and the rest (486 out of $514 ; 94.6 \%$ ) were downregulated (Figure $3 \mathrm{C}$ ). This suggests that NF was inclined to activate gene expression to cope with Sporisorium destruens stress.
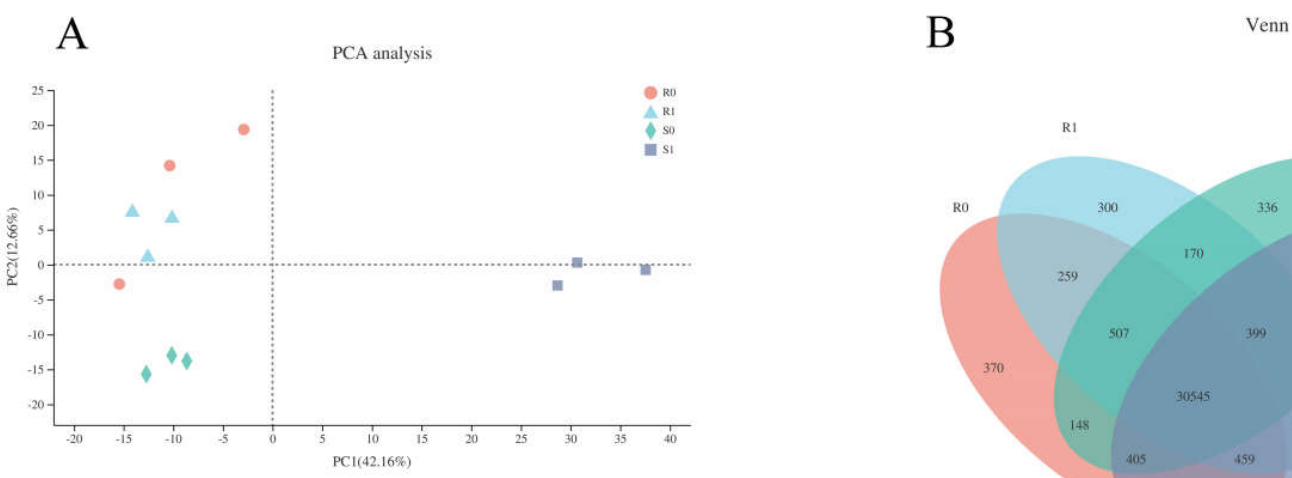

C Differential statistics
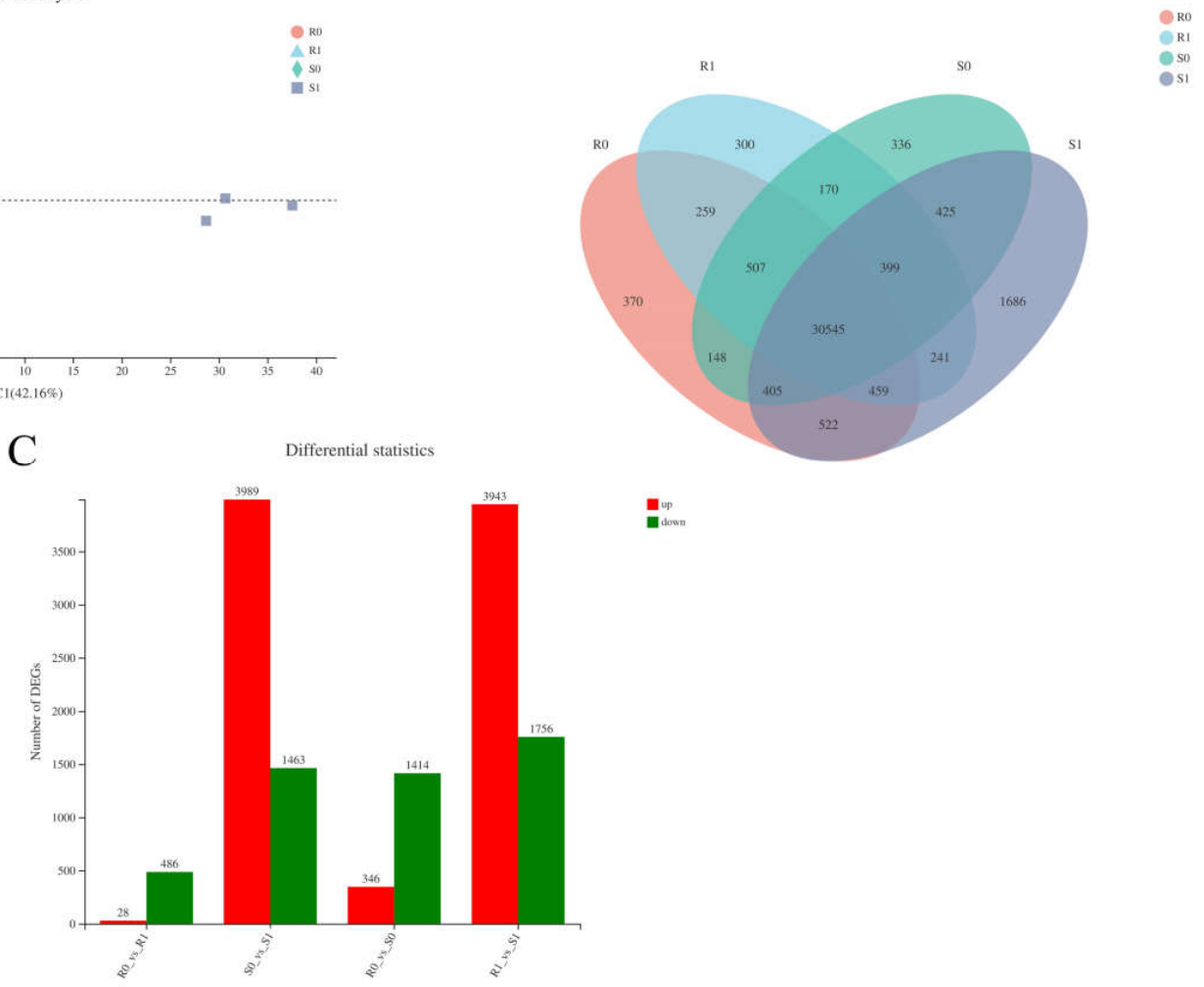

$\mathbf{m}_{\infty \times n}^{\infty}$

Figure 3. Analysis of transcriptional differences in four samples. (A) PCA analysis of four samples: the closer the sample, the higher the similarity. (B) Venn diagram of expressed genes of four samples. (C) The number of differentially expression genes (DEGs) in the different group.

\subsection{Gene Ontology (GO) Enrichment Analysis of DEGs}

A hypergeometric distribution was used to divide the DEGs into GO terms. BM and NF had 350 and 2517 enriched GO terms, respectively. Specifically, in BM, GO enrichment analysis included 82 molecular functions (MFs), 100 biological processes (BPs), and 1 cell 
component (CC), whereas the GO enrichment analysis of NF consisted of 151 MFs, 331 BPs, and 42 CCs (Supplementary Material file 3). Among the first twenty enriched GO terms in the two broomcorn millet varieties (Figure 4A), BM had only 12 terms that were significantly enriched $(p<0.05)$. Additionally, five terms were the same in the two varieties: oxidationreduction process (GO: 0055114), oxidoreductase activity (GO: 0016491), catalytic activity (GO: 0003824), iron ion binding (GO: 0005506), and dioxygenase activity (GO: 0051213). In the term type classification, the oxidation-reduction process belongs to $\mathrm{BP}$, and the remaining four items belong to MF, which showed that the MF of broomcorn millet was greatly affected after Sporisorium destruens inoculation. The catalytic activity was the term with the largest number of DEGs in both broomcorn millet varieties: compared with R0, 212 DEGs were enriched in R1; and 1412 DEGs were also enriched in S1 compared with S0.

A

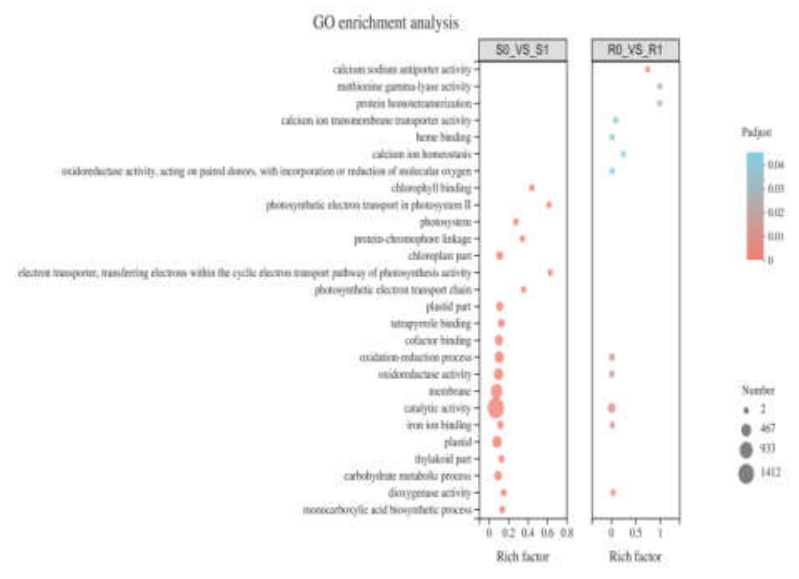

B

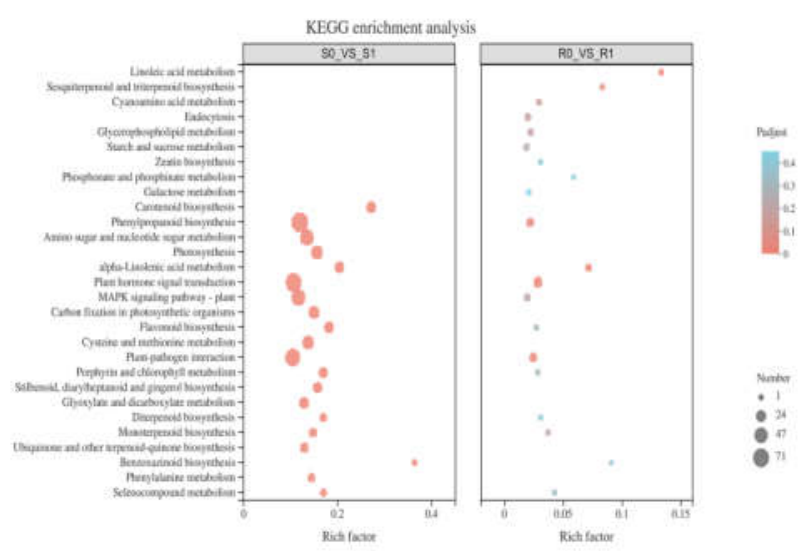

Figure 4. (A) GO enrichment analyses of the differentially expressed genes(DEGs) in NF (R0 vs. R1) and BM (S0 vs. S1) after the inoculation of Sporisorium destruens. (B) KEGG enrichment analysis of DEGs after the inoculation of Sporisorium destruens in R0 vs. R1 and S0 vs. S1.

\subsection{KEGG Enrichment Analysis}

The DEGs were mapped to the reference pathways in KEGG to investigate the interaction between Sporisorium destruens and broomcorn millet. KEGG enrichment analysis showed that 197 DEGs in R0 vs. R1, and 1286 DEGs in S0 vs. S1, were mapped onto 64 and 114 KEGG pathways, respectively, and there were 12 and 27 KEGG pathways that were significantly enriched, respectively $(p<0.05)$ (Supplementary Material file 4$)$. Figure $4 \mathrm{~B}$ plots the top 20 enrichment pathways of two broomcorn millet varieties, indicating that four significant enrichment pathways appeared in both varieties at the same time, namely phenylpropanoid biosynthesis, plant-pathogen interaction, plant hormone signal transduction, and alpha-linolenic acid metabolism pathways. In addition, pathways related to photosynthesis, photosynthesis-antenna proteins, and porphyrin and chlorophyll metabolism were significantly enriched in S0 vs. S1 $(p<0.05)$.

\subsection{DEGs Analysis of Different Leaf Types of Diseased Broomcorn Millet}

To further explore the interaction mechanism between diseased broomcorn millet and Sporisorium destruens, the transcriptomes of normal leaves on diseased plants (S1) and top cluster leaves (S2) were analysed (Figure 5A). Compared with S1, there were 4213 DEGs in S2, including 1456 up-regulated DEGs and 2757 down-regulated DEGs (Figure 5B). The number of down-regulated DEGs in S2 was 1.9-fold higher than that of up-regulated DEGs, which was contrary to the trend of S0 vs. S1. Different mechanisms in S0 vs. S1 and S1 vs. S2 were inferred from the differences in the changes between susceptible varieties. 
A

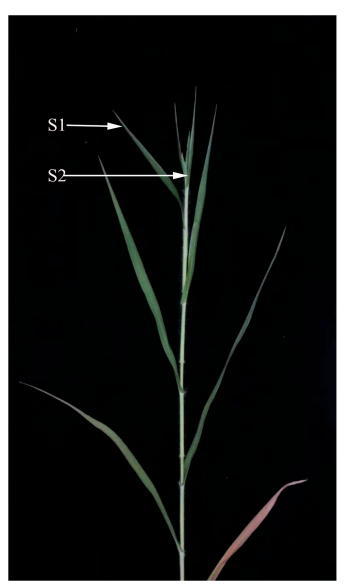

$\mathrm{C}$

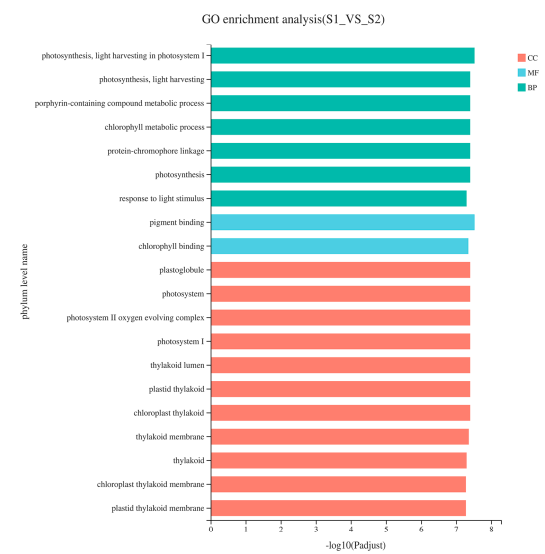

B

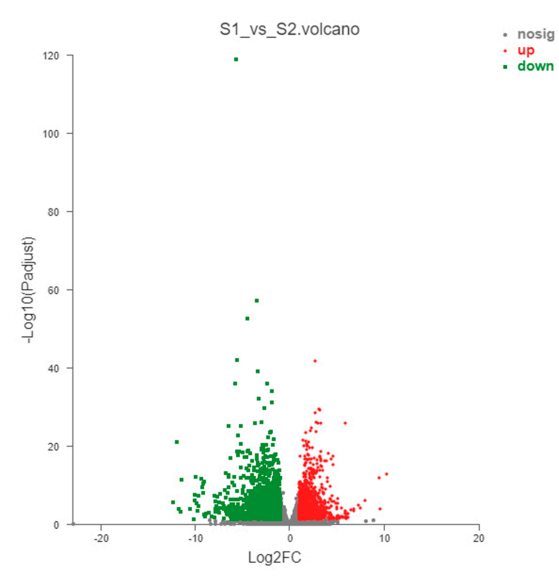

$\mathrm{D}$

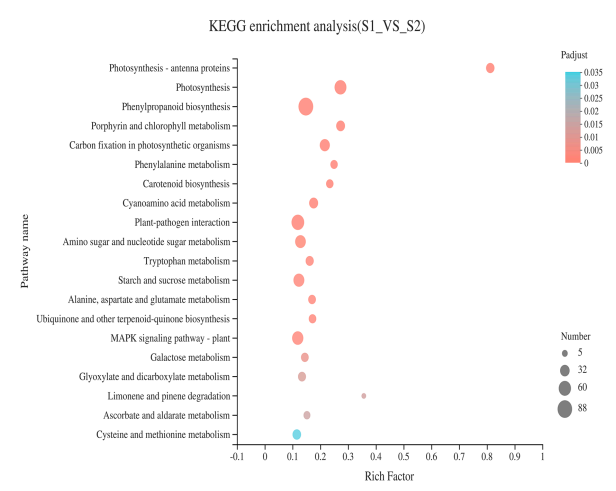

Figure 5. Difference between S1 and S2 in diseased plant. (A) The performance of S1 and S2 and their position on the diseased plant. (B) Distribution of DEGs in S2 compared to S1. (C) GO enrichment analysis of DEGs in S1 vs. S2. (D) KEGG enrichment analysis of DEGs in S1 vs. S2.

\subsection{GO and KEGG Enrichment Analyses of S1 vs. S2}

GO enrichment analysis showed that 3061 DEGs were distributed in 516 pathways in S1 vs. S2, of which 261 were significantly enriched (Supplementary Material file 3). Of these 261 pathways, 48,161 , and 52 were CC BP and MF terms, respectively. In the first 20 pathways enriched by GO, there were 7 BPs, 11 CCs, and 2 MFs (Figure 5C). Notably, among the 20 terms, those relating to photosynthesis accounted for a large proportion, such as photosynthesis (GO: 0015979), light harvesting in photosystem I (GO: 0009768) in BP, photosystem (GO: 0009521) in CC, and chlorophyll binding (GO: 0016168) in MF. Combined with the comprehensive analysis of the GO enrichment results in S0 vs. S1, we confirmed that photosynthesis played an important role in the interaction between Sporisorium destruens and broomcorn millet.

Compared with S1, there were 1478 DEGs involved in the KEGG pathways in S2, and the results showed that these DEGs were distributed in 120 pathways, of which 33 pathways were significantly enriched (Supplementary Material file 4). It was apparent that benzoic acid biosynthesis, photosynthesis, and photosynthesis-antenna pathways were the three most significantly enriched pathways (Figure 5D). Phenylpropane biosynthesis, photosynthesis, and plant-pathogen interaction pathways had the largest amount of enriched DEGs. KEGG enrichment analysis indicated that S0 vs. S1 showed different response mechanisms between Sporisorium destruens and broomcorn millet, although both involved photosynthesis. 


\subsection{DEGs Involved in Plant Hormone Signal Transduction Pathway}

In plant hormone signal transduction, the jasmonate ZIM (JAz)-domain-containing protein family is a key component of the jasmonic acid (JA) signalling pathway [28]. In the JAz family, 19 genes in five samples of two broomcorn millets were defined as DEGs (Figure 6). DEGs in the two varieties showed different trends: in BM, compared with R0, seven DEGs were down-regulated in R1; in S0 vs. S1, 18 DEGs were defined as being up-regulated; the 9 DEGs annotated in the JAz family of S1 vs. S2 included one downregulation and eight up-regulations. After Sporisorium destruens infection, the expression of JAz may be related to the resistance of broomcorn millet; in BM varieties, all the DEGs annotated in the JAz family were down-regulated, whereas in NF, most DEGs were upregulated. There were three DEGs annotated in the GH3 auxin-responsive gene family: one DEG was defined as up-regulated in S0 vs. S1, and two DEGs were down-regulated in S1 vs. S2. Furthermore, the expression of 12 genes in the auxin-responsive protein (IAA) family had changed: there were four up-regulated DEGs and one down-regulated DEG in S0 vs. S1, and two up-regulated DEGs and five down-regulated DEGs in S1 vs. S2 (Supplementary Material file 5). In the resistant variety of broomcorn millet, no DEGs were annotated in the IAA and GH3 families.

\section{Heatmap}

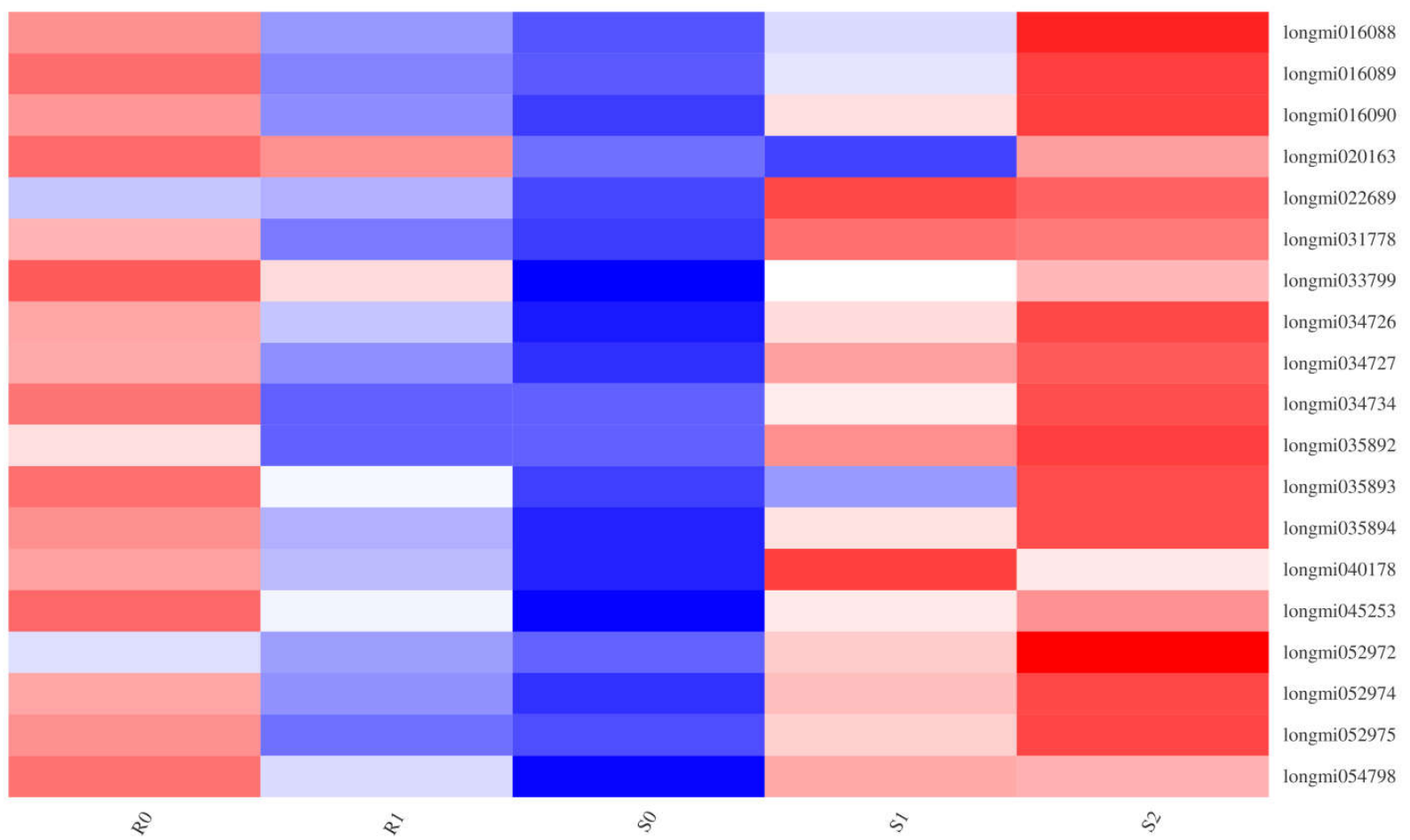

Figure 6. The heatmap about the correlation between DEGs enriched in JAz family and samples.

\subsection{Analysis of Enriched DEGs in the Plant-Pathogen Interaction Pathway}

Knowledge of the plant-pathogen interaction pathway is key to understanding the interactions between plants and pathogens. In the plant-pathogen interaction pathway of broomcorn millet and Sporisorium destruens, there were 13 DEGs in BM, 56 DEGs in S0 vs. S1, and 64 DEGs in S1 vs. S2, respectively (Supplementary Material file 6). Interestingly, most of the DEGs in BM were down-regulated (11 out of 13), whereas in NF the upregulated DEGs accounted for a larger proportion. Among the 56 DEGs enriched in S0 vs. S1, the number of up-regulated DEGs was 40. Furthermore, 43 DEGs were up-regulated in $\mathrm{S} 1$ vs. S2. These DEGs were mainly distributed in the calmodulin (CaM), calmodulin- 
like (CML), and 3-ketoacyl-CoA synthase (KCS) families. In addition, the expression of DEGs was affected in the calcium-dependent protein kinase (CDPK), respiratory burst oxidase homologue, and PTI families of NF, but this was not observed in BM (Figure 7A). DEGs enriched in plant-pathogen interaction pathways may affect cell wall reinforcement, stomatal closure, HR, defence-related gene induction, phytoalexin accumulation, miRNA production, suppression of plant HR, and defence responses. The leucine-rich repeat (LRR) transmembrane receptor kinase, flagellin-sensitive 2 (FLS2), is essential for flagellin perception [29]. There were three DEGs annotations in the FLS2 family, Longmi038613 and Longmi019029 were up-regulated in S0 vs. S1; Longmi038613 and Longmi019029 were down-regulated in S1 vs. S2. In this study, these DEGs may act as receptors or kinases in the HR, cell wall reinforcement, and stomatal closure.

A

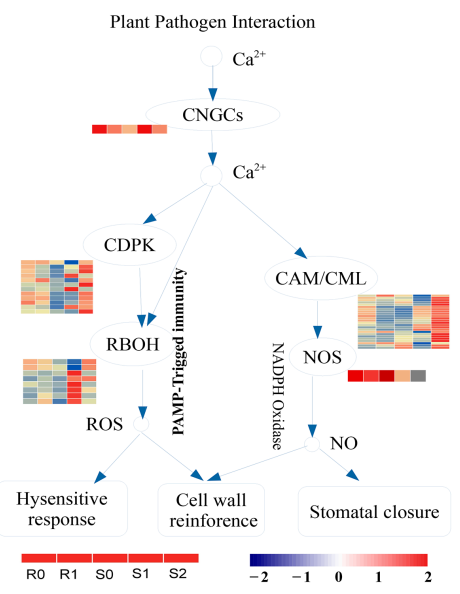

C

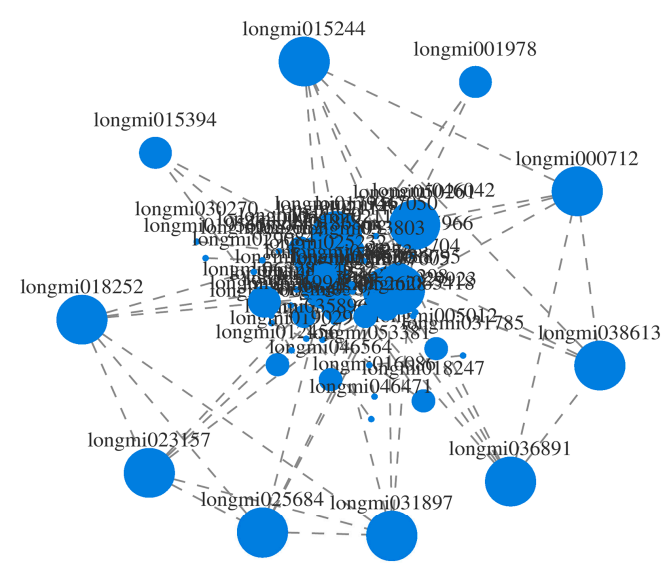

B

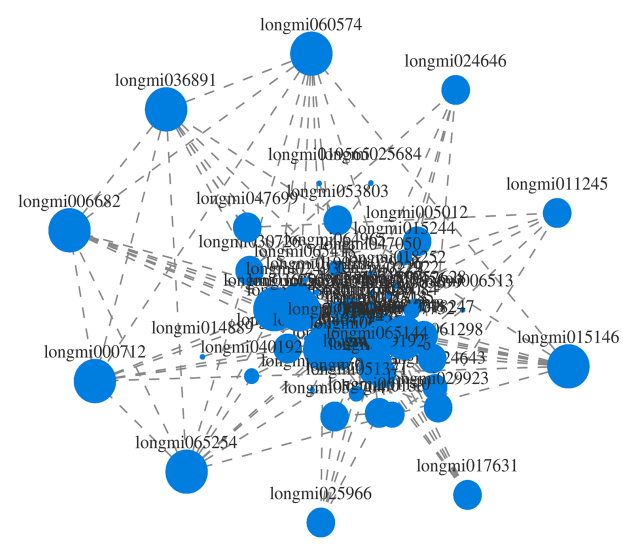

D

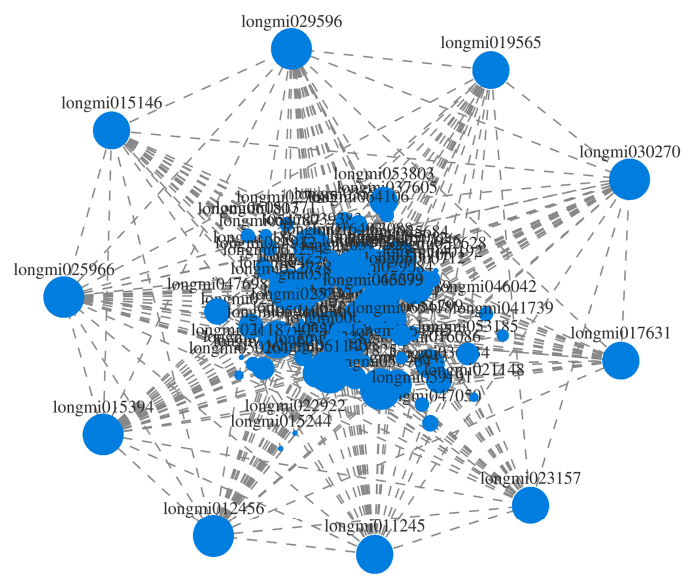

Figure 7. DEGs enriched in plant-pathogen interaction pathway. (A) DEGs related to $\mathrm{Ca}^{2+}$ in plant-pathogen interaction pathway. Expression related network diagram of DEGs enriched in plant-pathogen interaction pathway in R0 vs. R1 (B), S0 vs. S1 (C), and S1 vs. S2 (D).

2.10. Expression Correlation Analysis of Enriched DEGs in Plant-Pathogen Interaction Pathway

Expression correlation analysis is based on the correlation of gene expression and is used to identify key genes by analysing the connections between genes (Supplementary Material file 7). A correlation analysis of 95 DEGs in the plant-pathogen interaction pathway was carried out, and the DEGs with strong correlation was selected to 
draw the correlation network diagram (Figure 7B-D). Analysis of the top 10 genes with strong correlation in each group showed that both Longmi000712 and Longmi036891 were strongly correlated in R0 vs. R1, and in S0 vs. S1; these two genes may be related to the smut resistance of broomcorn millet. In the two groups of susceptible broomcorn millet, Longmi015394 and Longmi023157 showed a strong correlation, which may be related to the pathogenic mechanism of broomcorn millet. In addition to the above-mentioned genes, there were 8 DEGs with strong correlation in each treatment. These DEGs may be the reasons for the different resistances and pathogenesis of broomcorn millet.

\subsection{Real-Time PCR Verification of DEGs}

Ten DEGs were selected from the transcriptome results for RT-PCR verification of their reliability. The primer sequence is shown in Supplementary Material file 8. The RT-PCR verification and RNA-Seq data were roughly the same (Figure 8), indicating that the transcriptome results were reliable.
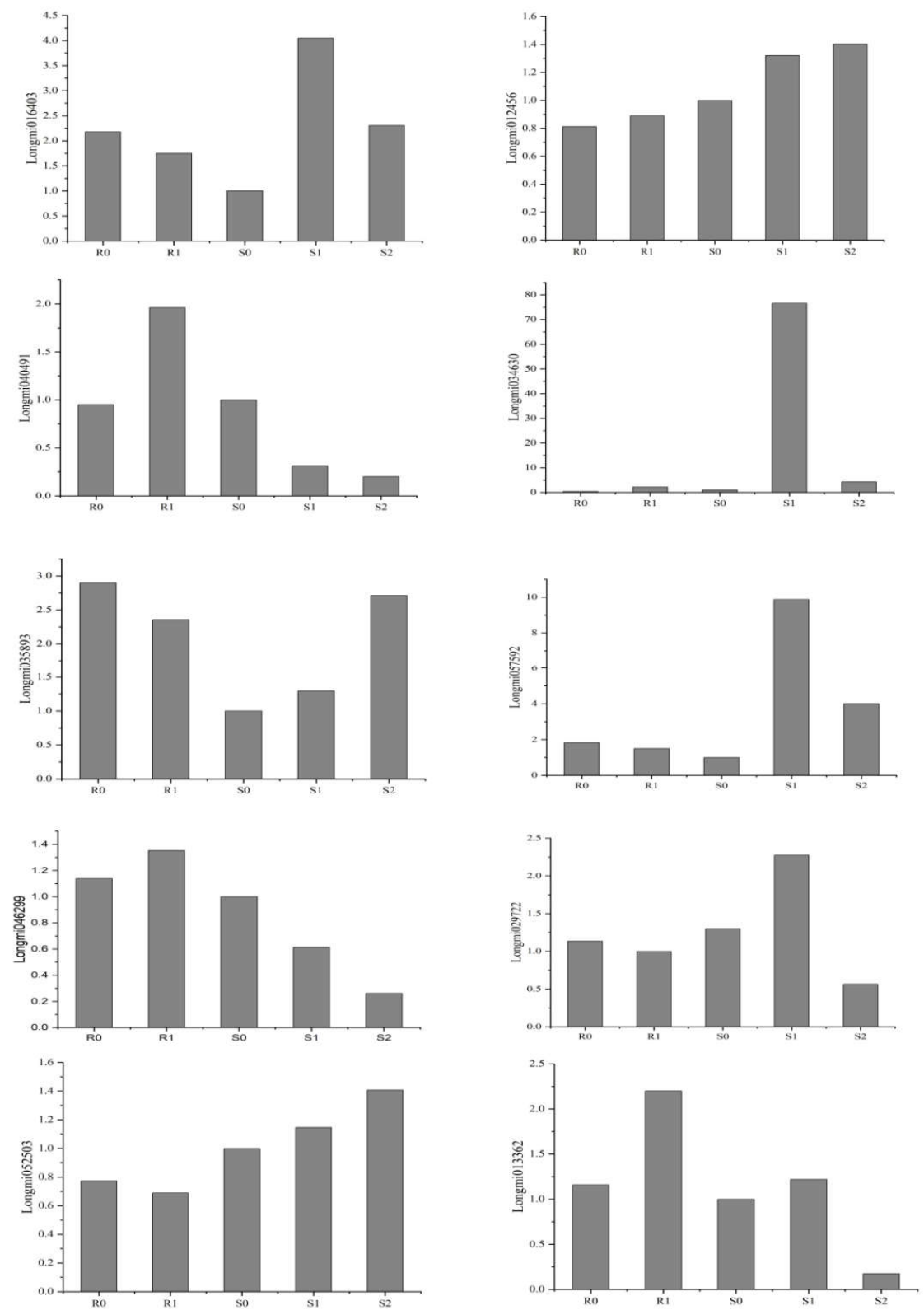

Figure 8. Validation of 10 genes by using RT-PCR. 


\section{Discussion}

Smut is a common crop disease which damages ears and grains and reduces economic output. Research on smut has mainly focused on crops such as rice, sugarcane, and maize $[1,30,31]$. This study used RNA-Seq to analyse the transcriptional changes in smutresistant and smut-susceptible broomcorn millet varieties after inoculation with Sporisorium destruens. Sequencing analysis identified 514 and 5452 DEGs in the resistant and susceptible cultivars, respectively. This result was consistent with the study by Cheng et al. [32], in which inoculation of Tainung 67 (resistant rice cultivar) and Zerawchanica karatals (susceptible rice cultivar) with Fusarium fujikuroi resulted in the appearance of 118 and 169 DEGs, respectively. Hence, susceptible varieties tend to activate gene expression in response to stress.

Plant immunity is a complicated process affected by extracellular or intracellular receptors that recognise PAMPs or effectors in PTI and effector-triggered immunity systems $[9,33]$. The defence response mechanisms and role of receptors have hardly been studied in broomcorn millet, but they have been reported in plants such as Arabidopsis, tomato, and rice [29,34-36]. In the plant-pathogen interaction pathway, there were 13, 56, and 64 DEGs in R0 vs. R1, S0 vs. S1, and S1 vs. S2, respectively. Compared with BM, NF expressed more DEGs after inoculation, and these DEGs may play an important role in the interaction between broomcorn millet and Sporisorium destruens.

$\mathrm{Ca}^{2+}$ acts as a second messenger in stress response signalling pathways [37], involving CaM, CaM-like (CML) proteins, and CDPKs [38]. Cyclic nucleotide-gated channels (CNGCs) reportedly play an important role in plant immunity, plant hormones, and response to stressors $[36,39]$. The CDPKs are crucial sensors of changes in $\mathrm{Ca}^{2+}$ concentration and have multiple roles in the stress tolerance of plants [40,41], while CaM plays a crucial role in plant defence signalling [42]. After inoculation with Sporisorium destruens, among the annotated DEGs were one gene in the CNGC family, 12 in the CDPK family, and 23 in the CaM/CML family (Supplementary Material file 9). In a study on canker disease in pitaya (Hylocereus polyrhizus) [43], infected tissue had two up-regulated unigenes and one down-regulated unigene in the CDPK family, and one up-regulated unigene annotated in the CaM/CML family. Herein, in the interaction between broomcorn millet and Sporisorium destruens, 12 annotated DEGs belonged to the CDPK family. Furthermore, eight DEGs in $\mathrm{S} 0$ vs. S1 were all up-regulated; 10 DEGs in S1 vs. S2, including eight up-regulated and two down-regulated. There were 23 annotated DEGs from the CaM/CML family, including two shared DEGs. Interestingly, the DEGs annotated in BM were all down-regulated, whereas all were up-regulated in NF. In the plant-pathogen interaction pathway, changes in the expression of these DEGs can affect cell wall reinforcement, stomatal closure, and HR, thereby affecting plant resistance.

In plants, disease resistance depends on the presence of complementary gene pairs in the host and pathogen. These are divided into resistance (R) genes and avirulence (Avr) genes, which can effectively curb the invasion of pathogens [44,45]. $R$ genes share some conserved domains, according to the arrangements of their functional domains, which can be grouped into five classes [46], based on the presence of an $\mathrm{N}$-terminal domain and a LRR domain. The LRR domain is responsible for specific pathogen recognition [47]. The resistance genes of the LRR domain have been reported in rice, wheat, and soybean [48-50], but not in broomcorn millet. Here, in the plant-pathogen interaction pathway, there were three annotated DEGs from the FLS2 family in the LRR domain, including one shared DEG: Longmi 038613 was defined as up-regulated DEG in S0 vs. S1, whereas it was defined as down-regulated DEG in S1 vs. S2 (Supplementary Material file 9). Different samples had different expression levels of Longmi038613, which requires verification to facilitate future research.

JA is an important phytohormone that regulates the defence responses of a plant $[51,52]$. In Arabidopsis, JA signalling has been negatively and positively associated with resistance against Fusarium graminearum [53]. Among the plant hormone signalling pathways, 18 DEGs in NF were up-regulated in the JAz family, and seven DEGs in BM were identified 
as being down-regulated. These results were consistent with a study on the response of broomcorn millet to the stress of Sporisorium destruens; the JA content increased after broomcorn millet infection [21]. It was inferred that invasion by Sporisorium destruens increased the JA content in the leaves of diseased broomcorn millet to resist Sporisorium destruens invasion. However, Cheng's research results showed that the genes associated with the JA biosynthetic process were up-regulated in the resistant cultivar, Selenio, and down-regulated in the susceptible Dorella cultivar [32], which was contrary to our result. Research on the role of JA in the interaction between broomcorn millet and smut needs further investigation.

When plant cells are subjected to adverse conditions, large amounts of reactive oxygen species (ROS) are produced [54], and the burst of ROS inhibits the process of chlorophyll electron transfer and photorespiration [55]. A high antioxidant capacity to scavenge the toxic ROS has been linked to stress tolerance [56]. The ROS in turn cause damage to lipids, and MDA content was taken as an indication of lipid peroxidation level [57]. Furthermore, changes in antioxidant protection enzymes have been reported in broomcorn millet $[21,58]$. Therefore, we measured SOD and POD activities in the leaves of each sample. Compared with $\mathrm{CK}, \mathrm{SOD}$ activity decreased in both varieties after inoculation. In the transcriptome results, there were no genes annotated in the SOD family of BM varieties, but in S0 vs. S1, three DEGs were annotated in the SOD family, all of which were down-regulated (Supplementary Material file 10). There was one down-regulated DEG annotation in S1 vs. S2. This trend was consistent with the measured SOD activity in NF leaves. In sugarcane leaves infected by orange rust, the SPAD index of reflecting chlorophyll content decreased with increasing rust rating [59]. In the photosynthetic report of Ustilago maydis infection of maize, the relative chlorophyll content of leaves was reduced after Ustilago maydis infection [60]. The SPAD index of broomcorn millet leaves after inoculation with Sporisorium destruens was lower than that of uninoculated controls; this may be the result of chlorophyll degradation by Sporisorium destruens infection. Furthermore, KEGG enrichment analysis indicated significantly enriched pathways related to photosynthesis, and chlorophyll metabolism. The role of photosynthesis in the interaction between broomcorn millet and Sporisorium destruens should be studied further.

\section{Materials and Methods}

\subsection{Plant Material, Sporisorium destruens Inoculation and Sample Collection}

The broomcorn millet varieties used in this study, namely Nianfeng No. 5 (NF) and BM, were provided by Northwest A\&F University (Yangling, China). BM was considered a smut-resistant variety; NF was considered a smut-susceptible variety. A two-year field survey showed that the incidence of Sporisorium destruens infection in BM and NF was $4.59 \%$ and $47.5 \%$, respectively. The spores of Sporisorium destruens were collected in the experimental field at Northwest A\&F University, dried in the shade at room temperature, surface-disinfected with $75 \%$ ethanol, and placed on an ultra-clean workbench. Then, the Sporisorium destruens spores were cut and collected with a sterilised blade, and the samples were placed in a kraft paper bag in a refrigerator at $4{ }^{\circ} \mathrm{C}$. It was removed from the refrigerator one day before use and kept at room temperature.

The experiment was carried out in a greenhouse at Northwest A\&F University in 2020. The soil and substrate (Pindstrup, Co., Ltd. Shanghai, China) were mixed in a 1:1 ratio. Seeds inoculated by saturation inoculation were mixed with Sporisorium destruens spores in a 20:1 mass ratio, representing the inoculation treatment. Uninoculated seeds served as the experimental control (CK). The seeds were planted in a black flowerpot (10 plants/pot) with a diameter of $20 \mathrm{~cm}$ and a height of $10 \mathrm{~cm}$, which were calculated based on the field density. The temperature and duration of illumination of the day/night cycles were set to $30^{\circ} \mathrm{C} / 18^{\circ} \mathrm{C}$ and $14 \mathrm{~h} / 10 \mathrm{~h}$, respectively. Relative humidity was constant at $60 \%$, and watering was performed every 1-2 days. The illumination intensity was set to $600 \mu \mathrm{mol} \mathrm{m}{ }^{-2} \mathrm{~s}^{-1}$. After showing symptoms at the heading stage, the lower leaves of the ear were collected: R0 (BM-CK), R1 (BM-inoculated), S0 (NF-CK), and S1 (NF-inoculated). 
In addition, the top cluster leaves of NF-diseased plants were collected and named S2. Collected leaf samples were stored in ultra-low-temperature refrigerators for Illumina deep sequencing, antioxidant enzyme activity assays, and RT-PCR validation.

\subsection{Phenotype Analysis of Broomcorn Millet}

After broomcorn millet heading, the ears of the diseased plant turn into small cluster leaves. At this stage, the phenotype of each treatment was evaluated. Five replicates were used to determine the differences in mean plant height $(\mathrm{cm})$, stem diameter $(\mathrm{mm})$, and node numbers of control plants and infected plants. Plant height and stem thickness were measured manually using a ruler and a digital calliper, respectively.

\subsection{Measurement of SOD Activity, POD Activity, MDA Content, and SPAD Index}

SOD and POD activities, as well as the MDA content, in the leaves under the ears of R0, R1, S0, S1, and S2, were determined using commercial detection kits (Solaibao Technology Co., Ltd., Beijing, China), according to the manufacturer's instructions. The SPAD index was determined using a non-invasive, handheld meter (SPAD-502, Konica Minolta, Tokyo, Japan).

\subsection{Transcriptome Sequencing of Broomcorn Millet Leaves}

Total RNA was extracted from the tissue using Plant RNA Purification Reagent for plant tissue, according to the manufacturer's instructions (Invitrogen, Carlsbad, CA, USA), and genomic DNA was removed using DNase I (Takara Bio, Kusatsu, Japan). Then RNA quality was determined using a 2100 Bioanalyzer (Agilent Technologies, Inc., Santa Clara, CA, USA) and quantified using the ND-2000 (NanoDrop Technologies, Inc., Wilmington, DE, USA).

The RNA-Seq library was prepared following the TruSeqTM RNA sample preparation kit from Illumina (San Diego, CA, USA) following the manufacturer's instructions. Briefly, messenger RNA was isolated according to the polyA selection method using oligo (dT) beads and then fragmented using fragmentation buffer. Second, double-stranded cDNA was synthesised using a SuperScript double-stranded cDNA synthesis kit (Invitrogen) with random hexamer primers (Illumina). The double-stranded cDNA was purified and ligated to adaptors for Illumina paired-end sequencing. Libraries were selected for cDNA target fragments of $300 \mathrm{bp}$ on $2 \%$ Low Range Ultra Agarose, followed by PCR amplification using Phusion DNA polymerase (New England Biolabs, Ipswich, MA, USA) for 15 PCR cycles. The resulting cDNA libraries were sequenced at Shanghai Majorbio Biopharm Technology Co., Ltd. (Shanghai, China) using the Illumina HiSeq xten/NovaSeq 6000 sequencing system.

\subsection{Transcriptome Analysis}

To identify the DEGs in broomcorn millets, transcript expression levels were calculated according to the transcripts per million reads method. RNA-Seq by ExpectationMaximization was used to quantify the gene abundance (http:/ / deweylab.biostat.wisc. $\mathrm{edu} / \mathrm{rsem} /$ (accessed on December 2020)). Differential expression analysis was performed using DESeq2, and genes with $|\log 2 \mathrm{FC}| \geq 1$ and $p \leq 0.05$ (DESeq2) were used as DEGs. Functional enrichment analysis of GO and KEGG was performed to identify the significant terms and pathways in terms of the Bonferroni-corrected $p$-value $\leq 0.05$, compared with the reference genome (http:/ /bigd.big.ac.cn/gwh/Assembly/131/show (accessed on November 2020)). GO enrichment terms and KEGG enrichment pathways were analysed using GOATOOLS (https:/ / github.com/tanghaibao/goatools (accessed on December 2020)) and KEGG Orthology-Based Annotation System (http://kobas.cbi.pku.edu.cn/download.php (accessed on December 2020)). 


\subsection{Real-Time PCR}

Selecting ten DEGs for real-time polymerase chain reaction (RT-PCR) to confirm the authenticity of the transcriptome results. These genes were annotated in pathways of photosynthesis, plant hormone signal transduction, phenylpropanoid biosynthesis, WRKY transcription factors, oxidation-reduction processes, plant-pathogen interactions, and alpha-linolenic acid metabolism. The primers for DEGs were designed and synthesised by Majorbio (Shanghai, China). Synthesis of cDNA was performed on HiScript Q RT SuperMix for qPCR (+gDNA wiper). RT-PCR was performed using the ABI7500 Real-time PCR System (Applied Biosystems, Waltham, MA, USA) with SYBR Premix Ex Taq II (Tli RNaseH Plus) and ROX plus (Takara Bio, Kusatsu, Japan). Expression levels of each gene were normalised relative to that of $\mathrm{S} 0$, and the fold-change in expression was calculated using the $2^{-\Delta \Delta C T}$ method.

\subsection{Statistical Analysis}

The physiological data obtained in the experiment were processed using the Statistical Package for Social Science (SPSS; SPSS Inc., Chicago, IL, USA) version 17.0, with statistical significance set at $p<0.05$. The chart was reprocessed using Origin version 2019 (OriginLab, Northampton, MA, USA).

\section{Conclusions}

In the two broomcorn millet varieties, NF stimulated more gene expression in response to Sporisorium destruens stress. KEGG enrichment analysis showed that plant hormone signal transduction, plant-pathogen interaction, and photosynthesis are important pathways in the interaction of broomcorn millet and Sporisorium destruens. After inoculation, 19 DEGs in the plant hormone signal transduction pathway were enriched in the JAz family of the JA signalling pathway. In the resistant variety (BM), the expression of JAz family DEGs decreased compared with CK; however, in the susceptible variety (NF), the DEGs of the JAz family after inoculation was up-regulated. In the plant-pathogen interaction pathway, the expression of DEGs from the CaM/CML, CDPK, and CNGC families in the $\mathrm{Ca}^{2+}$ signalling network can cause HR, cell wall reinforcement, and stomatal closure-adaptive responses to pathogen invasion. Finally, RT-PCR verification of 10 DEGs selected from the RNA-Seq transcriptome results showed that the data were reliable.

Supplementary Materials: The following are available online at https:/ /www.mdpi.com/article/10 .3390/ijms22179542/s1.

Author Contributions: Conceptualisation, F.J. and J.L.; methodology, E.W.; software, F.J. and E.W.; investigation, F.J.; resources, B.F.; writing—original draft preparation, F.J. and P.Y.; writing—review and editing, J.G., X.G. and B.F.; funding acquisition, B.F. All authors have read and agreed to the published version of the manuscript.

Funding: We acknowledge funding from National Industry Technology Projects of Foxtail Millet and Sorghum grant CARS-06-A26; and Shaanxi Province Industry Technology Projects of Coarse Cereals grant NYKJ-2018-YL1; Shaanxi Province Key R\&D Projects grant 2018TSCXL-NY-03-01.

Institutional Review Board Statement: Not applicable.

Informed Consent Statement: Not applicable.

Data Availability Statement: Not applicable.

Conflicts of Interest: The authors declare that there is no conflict of interests regarding the publication of this paper. 


\section{References}

1. Tang, J.; Chen, X.; Yan, Y.; Huang, J.; Luo, C.; Tom, H.; Zheng, L. Comprehensive transcriptome profiling reveals abundant long non-coding RNAs associated with development of the rice false smut fungus, Ustilaginoidea virens. Environ. Microbiol. 2021. [CrossRef] [PubMed]

2. McNeil, M.; Roberts, A.; Cockerell, V.; Mulholland, V. Real-time PCR assay for quantification of Tilletia caries contamination of UK wheat seed. Plant Pathol. 2004, 53, 741-750. [CrossRef]

3. Fan, J.; Yang, J.; Wang, Y.-Q.; Li, G.-B.; Li, Y.; Huang, F.; Wang, W.-M. Current understanding onVillosiclava virens, a unique flower-infecting fungus causing rice false smut disease. Mol. Plant Pathol. 2016, 17, 1321-1330. [CrossRef]

4. Zhou, Y.; Qu, Y.; Zhu, M.; Liu, J.; Wang, Y.; Song, H.; Feng, B. Genetic diversity and virulence variation of Sporisorium destruens isolates and evaluation of broomcorn millet for resistance to head smut. Euphytica 2016, 211, 59-70. [CrossRef]

5. Schilling, L.; Matei, A.; Redkar, A.; Walbot, V.; Doehlemann, G. Virulence of the maize smutUstilago maydisis shaped by organ-specific effectors. Mol. Plant Pathol. 2014, 15, 780-789. [CrossRef] [PubMed]

6. Wu, E.G.; Zhou, Y.; Zhu, M.Q.; Liu, J.J.; Gao, X.L.; Feng, B.L. Identification and biological characteristics of Sporisorium destruens in broomcorn millet. J. Plant Prot. Res. 2020, 47, 101-109. (In Chinese) [CrossRef]

7. Macho, A.P.; Zipfel, C. Plant PRRs and the Activation of Innate Immune Signaling. Mol. Cell 2014, 54, 263-272. [CrossRef] [PubMed]

8. Göhre, V.; Spallek, T.; Häweker, H.; Mersmann, S.; Mentzel, T.; Boller, T.; de Torres, M.; Mansfield, J.W.; Robatzek, S. Plant Pattern-Recognition Receptor FLS2 Is Directed for Degradation by the Bacterial Ubiquitin Ligase AvrPtoB. Curr. Biol. 2008, 18, 1824-1832. [CrossRef]

9. Thomma, B.P.; Nürnberger, T.; Joosten, M.H. Of PAMPs and Effectors: The Blurred PTI-ETI Dichotomy. Plant Cell 2011, 23, 4-15. [CrossRef] [PubMed]

10. Tian, W.; Hou, C.; Ren, Z.; Wang, C.; Zhao, F.; Dahlbeck, D.; Hu, S.; Zhang, L.; Niu, Q.; Li, L.; et al. A calmodulin-gated calcium channel links pathogen patterns to plant immunity. Nature 2019, 572, 131-135. [CrossRef] [PubMed]

11. Fanourakis, D.; Aliniaeifard, S.; Sellin, A.; Giday, H.; Körner, O.; Nejad, A.R.; Delis, C.; Bouranis, D.; Koubouris, G.; Kambourakis, E.; et al. Stomatal behavior following mid- or long-term exposure to high relative air humidity: A review. Plant Physiol. Biochem. 2020, 153, 92-105. [CrossRef] [PubMed]

12. Kaneda, T.; Taga, Y.; Takai, R.; Iwano, M.; Matsui, H.; Takayama, S.; Isogai, A.; Che, F.-S. The transcription factor OsNAC4 is a key positive regulator of plant hypersensitive cell death. EMBO J. 2009, 28, 926-936. [CrossRef] [PubMed]

13. Racapé, J.; Belbahri, L.; Engelhardt, S.; Lacombe, B.; Lee, J.; Lochman, J.; Marais, A.; Nicole, M.; Nürnberger, T.; Parlange, F.; et al. $\mathrm{Ca} 2^{+}$-dependent lipid binding and membrane integration of PopA, a harpin-like elicitor of the hypersensitive response in tobacco. Mol. Microbiol. 2005, 58, 1406-1420. [CrossRef]

14. Hepler, P.K.; Winship, L.J. Calcium at the Cell Wall-Cytoplast Interface. J. Integr. Plant Biol. 2010, 52, 147-160. [CrossRef]

15. Hunt, H.V.; Farah, B.; Olga, R.; Howe, C.J.; Jones, M.K.; Pat, H. Reticulate evolution in Panicum (Poaceae): The origin of tetraploid broomcorn millet, P. miliaceum. J. Exp. Bot. 2014, 65, 3165-3175. [CrossRef]

16. Liu, M.; Xu, Y.; He, J.; Zhang, S.; Wang, Y.; Lu, P. Genetic Diversity and Population Structure of Broomcorn Millet (Panicum miliaceum L.) Cultivars and Landraces in China Based on Microsatellite Markers. Int. J. Mol. Sci. 2016, 17, 370. [CrossRef] [PubMed]

17. Lu, H.; Zhang, J.; Liu, K.-B.; Wu, N.; Li, Y.; Zhou, K.; Ye, M.; Zhang, T.; Zhang, H.; Yang, X.; et al. Earliest domestication of common millet (Panicum miliaceum) in East Asia extended to 10,000 years ago. Proc. Natl. Acad. Sci. USA 2009, 106, 7367-7372. [CrossRef]

18. Bora, P.; Ragaee, S.; Marcone, M. Effect of parboiling on decortication yield of millet grains and phenolic acids and in vitro digestibility of selected millet products. Food Chem. 2019, 274, 718-725. [CrossRef] [PubMed]

19. Yue, H.; Wang, M.; Liu, S.; Du, X.; Song, W.; Nie, X. Transcriptome-wide identification and expression profiles of the WRKY transcription factor family in Broomcorn millet (Panicum miliaceum L.). BMC Genom. 2016, 17, 343-354. [CrossRef]

20. Dwivedi, S.L.; Upadhyaya, H.D.; Senthilvel, S.; Hash, C.T.; Fukunaga, K.; Diao, X.; Santra, D.; Baltensperge, D. Prasad M. Millets: Genetic and Genomic Resources; John Wiley \& Sons, Inc.: Hoboken, NJ, USA, 2012; Volume 35, pp. 247-375.

21. Wu, E.G.; Zhang, D.Z.; Liu, J.J.; Liu, Y.X.; Cao, X.L.; Feng, B.L. Study on responses of leaf protective enzyme and endogenous hormone to smut fungus stress in broomcorn millet. Plant Physiol. J. 2019, 6, 793-802. (In Chinese) [CrossRef]

22. Zhang, J.R.F.; Zhou, Y.; Yang, P.; Wang, P.K.; Gao, J.F.; Gao, X.L.; Feng, B.L. Study on physiological changes and correlation with resistance level to the head smut of broomcorn millet after an infection with Sphacelotheca destruen. J. China Agric. Univ. 2015, 20, 108-113. (In Chinese)

23. Liu, J.J.; Zhou, Y.; Zhang, P.P.; Chao, G.M.; Zhu, M.Q.; Feng, B.L. Efficacy of fungicides for control of head smut (Sporisorium destruens) in broomcorn millet. Acta Prataculturae Sin. 2016, 25, 132-141. (In Chinese) [CrossRef]

24. Liu, Y.X.; Zong, G.H.; Wu, E.G.; Zhang, D.Z.; Liu, L.; Wang, H.L.; Feng, B.L. Field identification and evaluation of smut resistant broomcorn millet resources based on agronomic traits. J. Northwest AF Univ. Nat. Sci. Ed. 2019, 12, 1-11. (In Chinese) [CrossRef]

25. Marioni, J.C.; Mason, C.E.; Mane, S.M.; Stephens, M.; Gilad, Y. RNA-seq: An assessment of technical reproducibility and comparison with gene expression arrays. Genome Res. 2008, 18, 1509-1517. [CrossRef] 
26. Qingzhu, H.; Chengjie, C.; Zhe, C.; Pengkun, C.; Yuewen, M.; Jingyu, W.; Jian, Z.; Guibing, H.; Jietang, Z.; Yonghua, Q. Transcriptomic Analysis Reveals Key Genes Related to Betalain Biosynthesis in Pulp Coloration of Hylocereus polyrhizus. Front. Plant Sci. 2016, 6, 1179-1192. [CrossRef]

27. Lai, Z.; Mengiste, T. Genetic and cellular mechanisms regulating plant responses to necrotrophic pathogens. Curr. Opin. Plant Biol. 2013, 16, 505-512. [CrossRef]

28. Thines, B.; Katsir, L.; Melotto, M.; Niu, Y.; Mandaokar, A.; Liu, G.; Nomura, K.; He, S.Y.; Howe, G.A.; Browe, J. JAz repressor proteins are targets of the SCF (COI1) complex during jasmonate signalling. Nature 2007, 448, 661-665. [CrossRef] [PubMed]

29. Chinchilla, D.; Bauer, Z.; Regenass, M.; Boller, T.; Felix, G. The Arabidopsis Receptor Kinase FLS2 Binds flg22 and Determines the Specificity of Flagellin Perception. Plant Cell 2006, 18, 465-476. [CrossRef] [PubMed]

30. Martinez, C.; Roux, C.; Dargent, R. Biotrophic Development of Sporisorium reilianum f. sp. Zeae in Vegetative Shoot Apex of Maize. Phytopathology 1999, 89, 247-253. [CrossRef]

31. Su, Y.; Wang, S.; Guo, J.; Xue, B.; Xu, L.; Que, Y. A TaqMan Real-Time PCR Assay for Detection and Quantification of Sporisorium scitamineum in Sugarcane. Sci. World J. 2013, 2013, 1-9. [CrossRef]

32. Cheng, A.-P.; Chen, S.-Y.; Lai, M.-H.; Wu, D.-H.; Lin, S.-S.; Chen, C.-Y.; Chung, C.-L. Transcriptome Analysis of Early Defenses in Rice against Fusarium fujikuroi. Rice 2020, 13, 1-15. [CrossRef]

33. Yasuda, S.; Okada, K.; Saijo, Y. A look at plant immunity through the window of the multitasking coreceptor BAK1. Curr. Opin. Plant Biol. 2017, 38, 10-18. [CrossRef]

34. Bent, A.; Kunkel, B.; Dahlbeck, D.; Brown, K.; Schmidt, R.; Giraudat, J.; Leung, J.; Staskawicz, B.J. RPS2 of Arabidopsis thaliana: A leucine-rich repeat class of plant disease resistance genes. Science 1994, 265, 1856-1860. [CrossRef]

35. Ron, M.; Avni, A. The Receptor for the Fungal Elicitor Ethylene-Inducing Xylanase Is a Member of a Resistance-Like Gene Family in Tomato. Plant Cell 2004, 16, 1604-1615. [CrossRef]

36. Nawaz, Z.; Kakar, K.U.; Saand, M.A.; Shu, Q.-Y. Cyclic nucleotide-gated ion channel gene family in rice, identification, characterization and experimental analysis of expression response to plant hormones, biotic and abiotic stresses. BMC Genom. 2014, 15, 1-18. [CrossRef] [PubMed]

37. Aldon, D.; Mbengue, M.; Mazars, C.; Galaud, J.-P. Calcium Signalling in Plant Biotic Interactions. Int. J. Mol. Sci. 2018, 19, 665. [CrossRef] [PubMed]

38. Ranty, B.; Aldon, D.; Cotelle, V.; Galaud, J.-P.; Thuleau, P.; Mazars, C. Calcium Sensors as Key Hubs in Plant Responses to Biotic and Abiotic Stresses. Front. Plant Sci. 2016, 7, 327. [CrossRef] [PubMed]

39. Moeder, W.; Urquhart, W.; Ung, H.; Yoshioka, K. The Role of Cyclic Nucleotide-Gated Ion Channels in Plant Immunity. Mol. Plant 2011, 4, 442-452. [CrossRef]

40. Chen, Y.; Zhou, X.; Chang, S.; Chu, Z.; Wang, H.; Han, S.; Wang, Y. Calcium-dependent protein kinase 21 phosphorylates 14-3-3 proteins in response to ABA signaling and salt stress in rice. Biochem. Biophys. Res. Commun. 2017, 493, 1450-1456. [CrossRef]

41. Pawełek, A.; Duszyn, M.; Świeżawska, B.; Szmidt-Jaworska, A.; Jaworski, K. Transcriptional response of a novel HpCDPK1 kinase gene from Hippeastrum $\times$ hybr. to wounding and fungal infection. J. Plant Physiol. 2017, 216, 108-117. [CrossRef]

42. Takabatake, R.; Karita, E.; Seo, S.; Mitsuhara, I.; Kuchitsu, K.; Ohashi, Y. Pathogen-Induced Calmodulin Isoforms in Basal Resistance Against Bacterial and Fungal Pathogens in Tobacco. Plant Cell Physiol. 2007, 48, 414-423. [CrossRef]

43. Xu, M.; Liu, C.-L.; Luo, J.; Qi, Z.; Yan, Z.; Fu, Y.; Wei, S.-S.; Tang, H. Transcriptomic de novo analysis of pitaya (Hylocereus polyrhizus) canker disease caused by Neoscytalidium dimidiatum. BMC Genom. 2019, 20, 10. [CrossRef]

44. Flor, H.H. Current Status of the Gene-For-Gene Concept. Annu. Rev. Phytopathol. 1971, 9, 275-296. [CrossRef]

45. Gu, B.; Cao, X.; Zhou, X.; Chen, Z.; Wang, Q.; Liu, W.; Chen, Q.; Zhao, H. The Histological, Effectoromic, and Transcriptomic Analyses of Solanum pinnatisectum Reveal an Upregulation of Multiple NBS-LRR Genes Suppressing Phytophthora infestans Infection. Int. J. Mol. Sci. 2020, 21, 3211. [CrossRef]

46. Miller, R.N.; Bertioli, D.J.; Baurens, F.C.; Santos, C.M.; Alves, P.C.; Martins, N.F.; Togawa, R.C.; Souza, M.T.; Pappas, G.J. Analysis of non-TIR NBS-LRR resistance gene analogs in Musa acuminata Colla: Isolation, RFLP marker development, and physical mapping. BMC Plant Biol. 2008, 8, 15. [CrossRef]

47. Meyers, B.C.; Dickerman, A.W.; Michelmore, R.W.; Sivaramakrishnan, S.; Sobral, B.W.; Young, N.D. Plant disease resistance genes encode members of an ancient and diverse protein family within the nucleotide-binding superfamily. Plant J. 1999, 20, 317-332. [CrossRef] [PubMed]

48. Yuan, B.; Zhai, C.; Wang, W.; Zeng, X.; Xu, X.; Hu, H.; Lin, F.; Wang, L.; Pan, Q. The Pik-p resistance to Magnaporthe oryzae in rice is mediated by a pair of closely linked CC-NBS-LRR genes. Theor. Appl. Genet. 2011, 122, 1017-1028. [CrossRef] [PubMed]

49. Jianyuan, L.; Xiaodong, W.; Lirong, Z.; Qingfang, M.; Na, Z.; Wenxiang, Y.; Daqun, L. A wheat NBS-LRR gene TaRGA19 participates in Lr19 -mediated resistance to Puccinia triticina. Plant Physiol. Biochem. 2017, 119, 1-8. [CrossRef] [PubMed]

50. Alekcevetch, J.C.; Passianotto, A.; Ferreira, E.; Santos, A.; Marcelino-Guimares, F.C. Genome-wide association study for resistance to the Meloidogyne javanica causing root-knot nematode in soybean. Theor. Appl. Genet. 2021, 134, 777-792. [CrossRef]

51. Yang, C.; Fernando, W. Hormonal Responses to Susceptible, Intermediate, and Resistant Interactions in the Brassica napusLeptosphaeria maculans Pathosystem. Int. J. Mol. Sci. 2021, 22, 4714. [CrossRef] [PubMed]

52. Liu, S.; Vargas, J.; Merewitz, E. Phytohormones associated with bacterial etiolation disease in creeping bentgrass. Environ. Exp. Bot. 2017, 133, 35-49. [CrossRef] 
53. Makandar, R.; Nalam, V.; Chaturvedi, R.; Jeannotte, R.; Sparks, A.; Shah, J. Involvement of Salicylate and Jasmonate Signaling Pathways in Arabidopsis Interaction with Fusarium graminearum. Mol. Plant-Microbe Interact. 2010, 23, 861-870. [CrossRef]

54. Dangl, J.L.; Jones, J. Plant pathogens and integrated defence responses to infection. Nature 2001, 411, 826-833. [CrossRef] [PubMed]

55. Wang, X.-S.; Han, J.-G. Changes of Proline Content, Activity, and Active Isoforms of Antioxidative Enzymes in Two Alfalfa Cultivars Under Salt Stress. Agric. Sci. China 2009, 8, 431-440. [CrossRef]

56. Chen, Y.C.; Fanourakis, D.; Tsaniklidis, G.; Aliniaeifard, S.; Yang, Q.Y.; Li, T. Low UVA intensity during cultivation improves the lettuce shelf-life, an effect that is not sustained at higher intensity. Postharvest Biol. Technol. 2021, 172, 111376. [CrossRef]

57. Hassanvand, F.; Nejad, A.R.; Fanourakis, D. Morphological and physiological components mediating the silicon-induced enhancement of geranium essential oil yield under saline conditions. Ind. Crop. Prod. 2019, 134, 19-25. [CrossRef]

58. Xu, B.Q.; Gao, X.L.; Gao, J.F.; Li, J.; Yang, P.; Feng, B.L. Transcriptome profiling using RNA-seq to provide insights into foxtail millet seedling tolerance to short-term water deficit stress induced by PEG-6000. J. Integr. Agric. 2019, 18, 2457-2471. [CrossRef]

59. Zhao, D.; Glynn, N.C.; Glaz, B.; Comstock, J.C.; Sood, S. Orange Rust Effects on Leaf Photosynthesis and Related Characters of Sugarcane. Plant Dis. 2011, 95, 640-647. [CrossRef]

60. Horst, R.J.; Engelsdorf, T.; Sonnewald, U.; Voll, L.M. Infection of maize leaves with Ustilago maydis prevents establishment of C4 photosynthesis. J. Plant Physiol. 2008, 165, 19-28. [CrossRef] 